\title{
THE POLAR CURVES OF PLANE ALGEBRAIC CURVES IN THE GALOIS FIELDS*
}

\author{
BY A. D. CAMPBELL
}

By imitating the proofs in Fine's College Algebra (pp. 460462) and Veblen and Young's Projective Geometry (vol. I, pp. 255-256) we can readily show that also in the Galois fields of order $p^{n}$ ( $p$ a prime integer) we have Taylor's expansion

$$
\begin{aligned}
& f(x+\lambda X, y+\lambda Y, z+\lambda Z) \\
& \equiv f(x, y, z)+\frac{\lambda}{1 !}\left(f_{x}^{\prime} X+f_{y}^{\prime} Y+f_{z}^{\prime} Z\right) \\
& \quad+\frac{\lambda^{2}}{2 !}\left(f_{x}^{\prime} X+f_{y}^{\prime} Y+f_{z}^{\prime} Z\right)^{(2)}+\cdots \\
& +\frac{\lambda^{r}}{r !}\left(f_{x}^{\prime} X+f_{y}^{\prime} Y+f_{z}^{\prime} Z\right)^{(r)}+\cdots+f(X, Y, Z)=0,
\end{aligned}
$$

where $\left(f_{x}^{\prime} X+f_{y}^{\prime} Y+f_{z}^{\prime} Z\right)^{(i)}$ is symbolic for an expression containing derivatives of the $i$ th order, and $f(x, y, z)=0$ is an algebraic curve of order $n$. In the above expansion we must take all the derivatives as though $p$ were not a modulus, cancel out common factors from numerators and denominators, and then set $p=0$.

The $r$ th polar of $(X, Y, Z)$ with respect to $f(x, y, z)=0$ is

$$
\frac{1}{r !}\left(f_{x}^{\prime} X+f_{y}^{\prime} Y+f_{z}^{\prime} Z\right)^{(r)}=0 .
$$

In particular the $r$ th polar of $(1,0,0)$ is $(1 / r !) \partial^{r} f(x, y, z) / \partial x^{r}$ $=0$. We suppose first of all that $n$ has the value

$$
\begin{aligned}
n=\alpha p^{m}+\beta p^{m-1}+\cdots+\gamma p^{2} & +\delta p+\epsilon, \\
& \neq 0, \quad p=\epsilon+\zeta, \quad \zeta \neq 0 .
\end{aligned}
$$

* Fresented to the Socict $y$, December 23, 1927. 
We can write the polars of $(1,0,0)$ by a sort of detached coefficients, underlining the coefficients that have $p$ as a factor, as follows:

$$
\begin{aligned}
& (1 / 1 !)[n, n-1, n-2, \cdots, \underline{n-\epsilon}, n-\epsilon-1, \\
& \left.\cdots, n-p-\epsilon, \cdots, n-p^{2}-\epsilon, \cdots, 3,2,1\right]=0 \text {, } \\
& (1 / 2 !)[n(n-1),(n-1)(n-2), \cdots,(n-\epsilon+1)(n-\epsilon) \text {, } \\
& (\underline{n-\epsilon})(n-\epsilon-1), \cdots,(n-p-\epsilon+1) \underline{(n-p-\epsilon)}, \\
& (n-p-\epsilon)(n-p-\epsilon-1), \cdots,\left(n-p^{2}-\epsilon+1\right) \\
& \text { - } \left.\left.\underline{\left(n-p^{2}-\epsilon\right.}\right), \underline{\left(n-p^{2}-\epsilon\right)}\left(n-p^{2}-\epsilon-1\right), \cdots, 3 \cdot 2,2 \cdot 1\right]=0 \text {, } \\
& {[1 /(\epsilon+1) !][n(n-1)(n-2) \cdots(\underline{n-\epsilon}),(n-1)(n-2) \cdots} \\
& \cdot(\underline{n-\epsilon})(n-\epsilon-1), \cdots,(\underline{n-\epsilon})(n-\epsilon-1) \cdots(n-2 \epsilon) \text {, } \\
& (n-\epsilon-1)(n-\epsilon-2) \cdots(n-2 \epsilon)(n-2 \epsilon-1), \cdots, \\
& (\epsilon+1) !]=0 \text {, } \\
& (1 / p !)[n(n-1) \cdots(\underline{n-\epsilon}) \cdots(n-p+1) \cdots, p !]=0 \text {, }
\end{aligned}
$$

where $(n-\lambda)(n-\lambda-1) \cdots(n-\lambda-i)$ stands for all the terms of the same $(n-\lambda-i-1)$ power, which then have this common factor in their coefficients. From the above polars we see that the $\epsilon$ th polar has at $(1,0,0)$ a tangent having $(\epsilon+1)$-point contact if $(1,0,0)$ is not on $f(x, y, z)=0$, otherwise a multiple point of order $\epsilon+1$. The $(\epsilon+1)$ th polar, $(\epsilon+2)$ th $, \cdots,(p-1)$ th polar all have multiple points of order $\epsilon+1$ at $(1,0,0)$. Similarly the $(p+\epsilon+1)$ th polar, $(p+\epsilon+2)$ th, $\cdots,(2 p-1)$ th have at $(1,0,0)$ multiple points of order $\epsilon+1$; also the $(2 p+\epsilon+1)$ th polar points of order $(3 p-1) \mathrm{h}, \cdots$, the $\left(\theta p^{i}+\cdots+\phi p+\epsilon+1\right)$ th polar points of order $\left(\theta p^{i}+\cdots+\phi p+p-1\right)$ th, etc. Moreover we note that if any one of the polar curves that have multiple points at $(1,0,0)$ is a curve of degree $\epsilon+1$, then this polar curve is degenerate. Thus for $p=2, n=2^{2}+1, \epsilon=1$, we find the $2 \mathrm{~d}$ 
polar is degenerate; for $p=3, n=3+1, \epsilon=1$, we find again the $2 \mathrm{~d}$ polar is degenerate.

If $n=\alpha p^{m}+\beta p^{m-1}+\cdots+\gamma p^{2}+\delta p$, i.e. $\epsilon=0$ in $n$, then all the polars of $(1,0,0)$ pass through $(1,0,0)$ whether or not this point lies on $f(x, y, z)=0$.

If $n<p$ we find no peculiarities like the above.

Syracuse University

\section{THE CHARACTERISTIC EQUATION OF A MATRIX*}

BY E. T. BROWNE

1. Introduction. Consider any square matrix $A$, real or complex, of order $n$. If $I$ is the unit matrix, $A-\lambda I$ is called the characteristic matrix of $A$; the determinant of the characteristic matrix is called the characteristic determinant of $A$; the equation obtained by equating this determinant to zero is called the characteristic equation of $A$; and the roots of this equation are called the characteristic roots of $A$. If $A$ happens to be a matrix of a particular type certain definite statements may be made as to the nature of its characteristic roots. For example, if $A$ is Hermitian its characteristic roots are all real; if $A$ is real and skewsymmetric, its characteristic roots are all pure imaginary or zero; if $A$ is a real orthogonal matrix, its characteristic roots are of modulus unity. However, if $A$ is not a matrix of some special type, no general statement can be made as to the nature of its characteristic roots. In 1900 Bendixson $\dagger$ proved that if $\alpha+i \beta$ is a characteristic root of a real matrix $A$, and if $\rho_{1} \geqq \rho_{2} \geqq \cdots \geqq \rho_{n}$ are the characteristic roots (all real) of the symmetric matrix $\frac{1}{2}\left(A+A^{\prime}\right)$, then $\rho_{1} \geqq \alpha \geqq \rho_{n}$. The extension to the case where the elements of $A$ are com-

\footnotetext{
* Presented to the Society, December 28, 1927.

$\dagger$ Bendixson, Sur les racines d'une équation fondamentale, Acta Mathematica, vol. 25 (1902), pp. 359-365.
} 